\title{
Time Periodic Perturbations of Quantum Systems
}

\author{
A. Kelemen \\ Institut für theoretische Physik der Technischen Universität Hannover
}

Z. Naturforsch. 34a, 1404-1409 (1979); received November 5, 1979

\begin{abstract}
We discuss a method of calculating the mean energy of a quantum system if the latter is subjected to time periodic perturbations. This, e.g., includes the possibility of determining shapes of spectral lines for an arbitrary distribution of nonperturbed energy levels. The method is studied on a system of order 2 whose spectral line is exactly lorentzian. We prove that the next to lowest approximation reproduces this form exactly.
\end{abstract}

\section{Introduction}

In quantum physics, we often consider quantum mechanical systems consisting of a set of levels that interact with time periodic perturbations. Such is the situation, for instance, of an atom upon which a periodic electromagnetic wave is incident. If the quantum electrodynamic effects are neglected, these systems are governed by linear differential equations with time periodic coefficients. Mathematical investigations of such differential equations set in with the basic paper by Floquet [1], and since then a huge number of articles treating this subject has appeared. For a review, see [2], pp. 55-59, and [3], pp. 78-81. A particularly suitable method, restricted to small perturbations, for the determination of the solutions and of characteristic exponents was developed by Cesari (see [2], pp. $68-72$, and [4]) where he also proves the convergence of the limit processes developed there.

From the quantum physicist's point of view, while the characteristic exponents might be relevant, the actual solutions are unobservable and therefore are less relevant. What is, for instance, measurable is the mean energy contained in the system, as it is defined by Eqs. (12) and (13). We remark that this mean energy is closely related to the shape of spectral lines if the perturbations are of electromagnetic nature. It would, therefore, be more advantageous to develop methods which enable one to calculate the observable quantities directly.

This is actually done in the following section where the observable quantity of interest will be the above mentioned mean energy. We achieve it by an infinite process whose quality must be

Reprint requests to Dr. A. Kelemen, Essenerstr. 37, D-6090 Rüsselsheim. examined. For such purposes, it is most suitable to consider quantum systems which can be exactly solved. A system of order 2 of the type (37) provides such an example where the associated spectral line is rigorously lorentzian. The necessary details are presented in the third section. The last section is reserved to a demonstration of the following interesting property: the calculation of the spectral line in the next to lowest order (according to the process devised in the second section) yields the exact result. This is quite surprising because it is independent of the strength of the perturbation.

\section{The Method}

Quantum mechanical systems we will be interested in are governed by the set of equations

$$
i \dot{c}_{i}(t)=\sum_{j} H_{i j}(t) c_{j}(t),
$$

where for $t>0$

$$
H(t+T)=H(t), \quad H^{+}(t)=H(t),
$$

$T$ being the period of the system. In (2) we use the matrix notation

$$
(H(t))_{i j}=H_{i j}(t), \quad\left(H^{+}(t)\right)_{i j}=H_{j i}^{*}(t),
$$

where the asterisk denotes the complex conjugate quantity. It is also convenient to introduce the vector notation for $c_{i}(t)$ : the symbol $|c(t)\rangle$ denotes the $n$-column vector with the components $c_{i}(t)$. We put

and

$$
(|c(t)\rangle)_{i}=c_{i}(t), \quad(\langle c(t)|)_{i}=c_{i} *(t)
$$

$$
\langle c(t) \mid c(t)\rangle=\sum_{i} c_{i} *(t) c_{i}(t) .
$$

For the sake of brevity, we shall write $|c\rangle$ for $|c(0)\rangle$. With the help of this notation, (1) can be

0340-4811/79 / 1200-1404 $\$ 01.00 / 0$. - Please order a reprint rather than making your own copy. 
written in the form

$$
i \frac{\mathrm{d}}{\mathrm{d} t}|c(t)\rangle=H(t)|c(t)\rangle .
$$

Let $G(t)$ denote the unique fundamental $n \times n$ matrix of the system $(6)$ with $G(0)=I(I$ the identity matrix of order $n$ ), then

$$
|c(t)\rangle=G(t)|c\rangle \text {. }
$$

The periodicity condition (2) implies now the basic relation

$$
|c(t+T)\rangle=G(t)|c(T)\rangle
$$

or, if combined with (7),

$$
G(t+T)=G(t) G(T) .
$$

We now assume that

$$
H(t)=E+V(t), \quad V(t)=0 \text { for } t<0,
$$

where the matrix elements of $E$,

$$
E_{i j}=E_{i} \delta_{i j},
$$

are supposed to be time independent. Since $V$ depends on time, the total energy is not conserved but we still can introduce the concept of the energy $\varepsilon$ embodied in the system as

$$
\varepsilon(t)=\sum_{i}\left|c_{i}(t)\right|^{2} E_{i}=\langle c(t)|E| c(t)\rangle .
$$

The mean amount of energy of the system is given by

$$
\bar{\varepsilon}=\lim _{t \rightarrow \infty} \frac{1}{t} \int_{0}^{t} \mathrm{~d} t^{\prime} \varepsilon\left(t^{\prime}\right) .
$$

Generally, $\bar{A}$ (or $\overline{A(t)}$ ) shall designate in the following the time mean value of $A(t)$ defined analogously to (13) where $A(t)$ is a function of time. We shall adopt this definition also for the case of $A(t)$ being a matrix whose matrix elements depend on time; then, of course, $\bar{A}$ is also a matrix.

It is obvious why the quantity defined by (13) is of interest: the spectral lines of emitting or absorbing systems are closely related to $\bar{\varepsilon}$. Indeed, what is measured is $\bar{\varepsilon}-E_{i}$ where $E_{i}$ is the energy of the system before the perturbation $V(t)$ has been switched on. Accordingly

$$
\varepsilon(t)=\left\langle c\left|G^{+}(t) E G(t)\right| c\right\rangle
$$

whence

$$
\bar{\varepsilon}=\left\langle c\left|\overline{G^{+}(t) E G(t)}\right| c\right\rangle .
$$

In general, however, it is not easy to evaluate (14 b) because of the limiting procedure in (13). On the other hand, if we specialize to systems with the property (2), we can fortunately calculate (14b) relatively simply. As the first step, we utilize (9) to get

$$
G(t)=G\left(t-T[t / T]_{G}\right) G^{[t / T]_{G}}(T),
$$

where $[a]_{G}$ stands for the gaussian symbol: for a real, $[a]_{G}$ designates the integer part of $a$,

$$
[a]_{G} \leqq a<[a]_{G}+1 .
$$

Here we see that whereas $t$ is arbitrary positive, the argument of the first $G$ matrix on the right side of (15) fulfills the inequality

$$
0 \leqq t-T[t / T]_{G}<T .
$$

This relation reveals the fundamental property of the theory: it suffices completely to know $G(t)$ for $0 \leqq t<T$ to calculate (14b), for which, in the second step, we rewrite (13):

$\bar{\varepsilon}=\lim _{N \rightarrow \infty} \frac{1}{N T} \int_{0}^{N T} \mathrm{~d} t \varepsilon(t)=\lim _{N \rightarrow \infty} \frac{1}{N T} \sum_{n=0}^{N-1} \int_{n T}^{(n+1) T} \mathrm{~d} t \varepsilon(t)$.

The third step consists in diagonalizing the matrix $G(T)$ :

$$
G_{i j}(T)=\sum_{k} U_{k i}^{*} g_{k} U_{k j},
$$

where use has been made of the fact that the matrix $G(T)$ (as well as $G(t)$ ) is unitary. This property is a consequence of the hermiticity of the matrix $H(t)$, Eq. (2); for more details see [5], pp. 722-724.

Now, since the matrix $U$ in (19) is unitary, we easily find $\left([t / T]_{G}\right.$ is integer)

$$
G_{i j}^{[t / T]_{G}}(T)=\sum_{k} U_{k i}^{*} g_{k}^{[t / T]_{G}} U_{k j} .
$$

To evaluate (18), we make use of (14a) into which (15) and (20) have to be inserted. Consequently,

$$
\begin{aligned}
\varepsilon(t)= & \sum_{i j}\left\langle c\left|U^{+}\right| i\right\rangle g_{i}^{*[t / T]_{G}} g_{j}^{[t / T]_{G}}\langle j|U| c\rangle \\
& \cdot\langle i| U G^{+}\left(t-T[t / T]_{G}\right) \\
& \cdot E G\left(t-T[t / T]_{G}\right) U^{+}|j\rangle,
\end{aligned}
$$

where the vectors $|i\rangle$ are supposed to form a basis of the complex $n$-dimensional vector space, in which the matrix $H(t)$ acts. This form of $\varepsilon(t)$ used 
in (18) gives rise to the following equation:

$$
\begin{aligned}
\bar{\varepsilon}= & \lim _{N \rightarrow \infty} \sum_{i j} \frac{1}{N T} \sum_{n=0}^{N-1} \int_{n T}^{(n+1) T} \mathrm{~d} t\left\langle c\left|U^{+}\right| i\right\rangle \\
& \cdot g_{i}^{*[t / T]_{G}} g_{j}^{[t / T]_{G}}\langle j|U| c\rangle \\
& \cdot\langle i| U G^{+}\left(t-T[t / T]_{G}\right) \\
& \cdot E G\left(t-T[t / T]_{G}\right) U^{+}|j\rangle .
\end{aligned}
$$

It is natural to introduce the transformation

$$
t-n T \rightarrow t,
$$

which has the consequence for the new $t$ that

$$
0 \leqq t<T, \quad[(t+n T) / T]_{G}=n .
$$

Hence

$$
\begin{aligned}
\bar{\varepsilon}= & \sum_{i j}\left\langle c\left|U^{+}\right| i\right\rangle\langle j|U| c\rangle T^{-1} \\
& \cdot \int_{0}^{T} \mathrm{~d} t\left\langle i\left|U G^{+}(t) E G(t) U^{+}\right| j\right\rangle \\
& \cdot \lim _{N \rightarrow \infty} N^{-1} \sum_{n=0}^{N-1} g_{i}^{* n} g_{j}^{n} .
\end{aligned}
$$

This version is undoubtedly superior to $(14 \mathrm{~b})$ since the integration extends over a finite region and the limiting procedure can be done independently.

To do the latter we group the eigenvalues $g_{i}$ in sets $A_{1}, A_{2}, \ldots$, which are composed of equal $g_{i}$. If we realize that $g_{i}$ are eigenvalues of a unitary matrix then we have

$$
g_{i}=e^{i \varphi_{i}}
$$

which implies

$$
\lim _{N \rightarrow \infty} N^{-1} \sum_{n=0}^{N-1} g_{i}^{* n} g_{j}^{n}= \begin{cases}1 & \text { for } g_{i}=g_{j}, \\ 0 & \text { otherwise }\end{cases}
$$

We thus finally arrive at

$$
\begin{aligned}
\bar{\varepsilon}= & \sum_{i} \sum_{k, l \in A_{i}}\left\langle c\left|U^{+}\right| k\right\rangle\langle l|U| c\rangle \\
& \cdot T^{-1} \int_{0}^{T} \mathrm{~d} t\left\langle k\left|U G^{+}(t) E G(t) U^{+}\right| l\right\rangle .
\end{aligned}
$$

Should it happen that all $g_{i}$ are distinct then (28) obviously reduces to

$$
\begin{aligned}
\bar{\varepsilon}= & \sum_{i}\left|\left\langle c\left|U^{+}\right| i\right\rangle\right|^{2} T^{-1} \\
& \cdot \int_{0}^{T} \mathrm{~d} t\left\langle i\left|U G^{+}(t) E G(t) U^{+}\right| i\right\rangle,
\end{aligned}
$$

which will play a particular role in our further considerations.

How to evaluate (29) in practice? We have to determine the matrices $G(t)$ and $U$. For the former we go back to the Dyson expansion [6] writing

$$
G(t)=G_{0}(t) D(t),
$$

where

$$
G_{0}(t)=e^{-i E t}
$$

and

$$
\begin{aligned}
D(t)= & I+(-i) \int_{0}^{t} \mathrm{~d} t^{\prime} e^{i E t^{\prime}} V\left(t^{\prime}\right) e^{-i E t^{\prime}} \\
& +(-i)^{2} \int_{0}^{t} \mathrm{~d} t^{\prime} e^{i E t^{\prime}} V\left(t^{\prime}\right) e^{-i E t^{\prime}} \\
& \cdot \int_{0}^{t^{\prime}} \mathrm{d} t^{\prime \prime} e^{i E t^{\prime \prime}} V\left(t^{\prime \prime}\right) e^{-i E t^{\prime \prime}}+\cdots .
\end{aligned}
$$

However, it is pointed out in [2] and [4] that this classical series has drawbacks (e.g. it may give polynomial approximations of functions which are periodic). It is therefore necessary to modify (32) so as to avoid these drawbacks, and in [2] and [4] one such a modification (and a proof of its convergence) can be found. In the following, we present another modification of (32) but are unable to give a rigorous proof of its convergence. An investigation on a computer has, however, shown that the process converges if applied to a system of order 2 (explained in the third section), the convergence being independent of the strength of $V$. We shall now briefly expound this process.

It goes without saying that the unitarity property of $D(t)$ is lost if we retain only several lowest order terms in the expansion (32); let us designate this approximate $D(t)$ as $D^{\prime}(t)$. In order to restore the unitarity, we could e.g. proceed as follows: first diagonalize $D^{\prime}(t)$

$$
D^{\prime}(t)=W^{+}(t) d(t) W(t) .
$$

Secondly, modify the diagonal elements of the diagonal matrix $d(t)$ to be in accordance with (26):

$$
\overline{d_{i}(t)}=d_{i}(t) /\left|d_{i}(t)\right|,
$$

and approximate

$$
D(t) \approx \tilde{D}(t)=W^{+}(t) \overline{d(t)} W(t) .
$$

Clearly, $\tilde{D}(t)$ is unitary and we use it in place of $D(t)$ in $(30)$,

$$
G(t) \approx \tilde{G}(t)=G_{0}(t) \tilde{D}(t) .
$$

The matrix $U$ is obtained from (21) on replacing $G$ by $\tilde{G}$.

Before we apply these formulas, we shall first discuss a soluble model for which $(14 b)$ can be 
evaluated exactly. This is of course important because it is possible only for such cases to recognize the quality of our approximation scheme $(29)-(36)$.

\section{A System of Order 2 (Zener [7])}

It was probably first found by Zener [7] that a system of order 2 is particularly suitable for approximating complex physical processes (such as non-adiabatic crossing of energy levels). This author realized that a system of order 2

$$
\begin{aligned}
& i \dot{c}_{1}(t)=H_{11} c_{1}(t)+H_{12} c_{2}(t), \\
& i \dot{c}_{2}(t)=H_{21} c_{1}(t)+H_{22} c_{2}(t),
\end{aligned}
$$

$H_{11}, \ldots, H_{22}$ being functions of time $t$, admits rigorous solutions for various interesting physical situations. He showed, for example, that the motion of two atoms influences the transition between two electronic states (all other states being neglected) in a way described by Equations (37). Assuming that the transition region is small, Zener introduced the additional simplification that

$$
H_{11}-H_{22} \sim t, \quad \dot{H}_{12}=\dot{H}_{21}=0 .
$$

It is then possible to solve (37) exactly because (37) can be reduced to the Weber equation.

Another application of (37) is provided by considering 2 electronic states of an atom which is subjected to a periodic electromagnetic field. We may then assume that

$H_{11}=E_{1}, \quad H_{22}=E_{2}, \quad H_{12}=H_{21}^{*}=V e^{i \omega t}$,

where $E_{1}$ and $E_{2}$ are energies of the unperturbed electronic states. It is now not difficult to demonstrate that the solution of (37) can be given in a closed form. Making use of (7) we arrive at

$$
\begin{aligned}
G_{11}(t)= & \left(E_{-} 2+4|V|^{2}\right)^{-1 / 2} \exp \left(-\frac{1}{2} i t E^{-}\right) \\
& \cdot\left\{i E_{-} \sin \left[\frac{1}{2} t\left(E_{-}{ }^{2}+4|V|^{2}\right)^{1 / 2}\right]\right. \\
& +\left(E_{-} 2+4|V|^{2}\right)^{1 / 2} \\
& \left.\cdot \cos \left[\frac{1}{2} t\left(E_{-}{ }^{2}+4|V|^{2}\right)^{1 / 2}\right]\right\}, \\
G_{12}(t)= & -2 i V\left(E_{-} 2+4|V|^{2}\right)^{-1 / 2} \\
& \cdot \exp \left(-\frac{1}{2} i t E^{-}\right) \sin \left[\frac{1}{2} t\left(E_{-}{ }^{2}+4|V|^{2}\right)^{1 / 2}\right], \\
G_{21}(t)= & -2 i V^{*}\left(E_{-}{ }^{2}+4|V|^{2}\right)^{-1 / 2} \\
& \cdot \exp \left(-\frac{1}{2} i t E^{+}\right) \sin \left[\frac{1}{2} t\left(E_{-}{ }^{2}+4|V|^{2}\right)^{1 / 2}\right], \\
G_{22}(t)= & \left(E_{-} 2+4|V|^{2}\right)^{-1 / 2} \exp \left(-\frac{1}{2} i t E^{+}\right) \\
& \cdot\left\{-i E_{-} \sin \left[\frac{1}{2} t\left(E_{-}{ }^{2}+4|V|^{2}\right)^{1 / 2}\right]\right. \\
& +\left(E_{-}{ }^{2}+4|V|^{2}\right)^{1 / 2} \\
& \left.\cdot \cos \left[\frac{1}{2} t\left(E_{-}{ }^{2}+4|V|^{2}\right)^{1 / 2}\right]\right\}
\end{aligned}
$$

where

$$
\begin{aligned}
& E_{ \pm}=E_{2}-E_{1} \pm \omega, \\
& E^{ \pm}=E_{2}+E_{1} \pm \omega .
\end{aligned}
$$

For the sake of convenience, we introduce the matrix $T$

$$
T=\overline{G^{+}(t) E G(t)} .
$$

To calculate the mean energy $\bar{\varepsilon}$ of this two-level system, we may use $(14 \mathrm{~b})$. The calculation of matrix elements of $T$ is now straight-forward:

$$
\begin{aligned}
& T_{11}=E_{1}+\frac{2|V|^{2}\left(E_{2}-E_{1}\right)}{E_{-}{ }^{2}+4|V|^{2}}, \\
& T_{22}=E_{2}+\frac{2|V|^{2}\left(E_{1}-E_{2}\right)}{E_{-}{ }^{2}+4|V|^{2}},
\end{aligned}
$$

and

$$
T_{12}=T_{21}^{*}=\frac{V E_{-}\left(E_{2}-E_{1}\right)}{E_{-}{ }^{2}+4|V|^{2}} .
$$

Assume that the system at $t=0$ may be characterized by

$$
c_{1}=1, \quad c_{2}=0 \quad\left(E_{1}<E_{2}\right) .
$$

Substituting $(46)-(48)$ into $(14 \mathrm{~b})$ we obtain

$$
\bar{\varepsilon}-E_{1}=\frac{2|V|^{2}\left(E_{2}-E_{1}\right)}{E_{-}{ }^{2}+4|V|^{2}},
$$

where (45) has been used. This is the famous Lorentz formula ([5], pp. 994-1001) for the shape of a spectral line.

The Zener model (37) provides valuable information concerning periodic perturbations of atomic systems. Our main aim, however, is to apply the results of the preceding section to the present system of order 2 . We shall prove that this method reproduces (50) exactly if in expansion (32) the two leading terms are retained.

\section{The Lowest Order Calculation of the Matrix I}

As stated above we wish to focus our attention on the following problem: Let

$$
E=\left(\begin{array}{cc}
E_{1} & 0 \\
0 & E_{2}
\end{array}\right), \quad V(t)=\left(\begin{array}{cc}
0 & V e^{i \omega t} \\
V^{*} e^{-i \omega t} & 0
\end{array}\right) .
$$

From (32) we obtain

$$
D^{\prime}(t)=I-i \int_{0}^{t} \mathrm{~d} t^{\prime} e^{i E t^{\prime}} V\left(t^{\prime}\right) e^{-i E t^{\prime}},
$$


where only first order terms in $V$ have been retained. Using the approximation (52) it is possible to derive $(46)-(48) . G(t)$ of $(29)$ is to be replaced by $\widetilde{G}(t)$ $(\tilde{G}(t)$ is constructed via $(52)$ and $(33)-(36))$.

Comparing (14b) and (45) with (29) we obtain the following expression for $T$ :

$$
\begin{aligned}
T= & \sum_{i} U^{+}|i\rangle T^{-1} \\
& \cdot \int_{0}^{T} \mathrm{~d} t\left\langle i\left|U G^{+}(t) E G(t) U^{+}\right| i\right\rangle\langle i| U .
\end{aligned}
$$

Using $(30)-(36)$ of Sect. 2 we may now evaluate (52). In view of (51) we obtain

$$
D^{\prime}(t)=\left(\begin{array}{cc}
1 & i \mathscr{V}(t) \\
i \mathscr{V} *(t) & 1
\end{array}\right)
$$

where

$$
\mathscr{V}(t)=\frac{1}{i} \frac{V}{E_{-}}\left(e^{-i E_{-} t}-1\right) .
$$

In order to restore unitarity of $D^{\prime}(t)$ we use $(33)-(35)$ so that

$$
\tilde{D}(t)=\left[1+|\mathscr{V}(t)|^{2}\right]^{-1 / 2} D^{\prime}(t) .
$$

Therefore, according to (36), the corrected fundamental matrix $\widetilde{G}(t)$ becomes

$$
\begin{aligned}
\tilde{G}(t)= & {\left[1+|\mathscr{V}(t)|^{2}\right]^{-1 / 2} \exp \left[-\frac{1}{2} i t\left(E_{1}+E_{2}\right)\right] } \\
& \times\left(\begin{array}{cc}
\exp \left[-\frac{1}{2} i t\left(E_{1}-E_{2}\right)\right] & i \exp \left[-\frac{1}{2} i t\left(E_{1}-E_{2}\right)\right] \mathscr{V}(t) \\
i \exp \left[-\frac{1}{2} i t\left(E_{2}-E_{1}\right)\right] \mathscr{V} *(t) & \exp \left[-\frac{1}{2} i t\left(E_{2}-E_{1}\right)\right]
\end{array}\right)
\end{aligned}
$$

and a straight-forward calculation confirms the unitarity of $\widetilde{G}(t)$. $\widetilde{G}(t)$ is now used in place of $G(t)$ in (53) and the quantity

$$
\begin{aligned}
\tilde{G}^{+}(t) E \tilde{G}(t)=\left[1+|\mathscr{V}(t)|^{2}\right]^{-1} \\
\quad \times\left(\begin{array}{cc}
E_{1}+E_{2}|\mathscr{V}(t)|^{2} & i\left(E_{1}-E_{2}\right) \mathscr{V}(t) \\
i\left(E_{2}-E_{1}\right) \mathscr{V} *(t) & E_{1}|\mathscr{V}(t)|^{2}+E_{2}
\end{array}\right)
\end{aligned}
$$

may be derived, which has a simple structure.

Once the expression $\tilde{G}^{+}(t) E \widetilde{G}(t)$ has been obtained the unknown matrix $U$ may now be derived. The matrix $U$ is defined by (19) with $G(T)$ replaced by $\widetilde{G}(T)$. Now, $\widetilde{G}(T)$ can be written in the form

$$
\begin{aligned}
\tilde{G}(T)= & {\left[1+|\mathscr{V}(T)|^{2}\right]^{-1 / 2} } \\
& \cdot \exp \left[-\frac{1}{2} i T\left(E_{1}+E_{2}\right)\right] K,
\end{aligned}
$$

where the matrix $K$ has the structure

$$
K=\left(\begin{array}{cc}
a & b \\
-b^{*} & a^{*}
\end{array}\right)
$$

$a$ and $b$ being complex numbers. Therefore, both $\tilde{G}(T)$ and $K$ can be diagonalized by the same unitary matrix $U$. We may then infer from $(60)$

$U=2^{-1 / 2}\left[\operatorname{Im}(a)^{2}+|b|^{2}\right]^{-1 / 4}\left(\begin{array}{cc}A_{+} & -i \frac{|b|}{b^{*}} A_{-} \\ A_{-} & i \frac{|b|}{b^{*}} A_{+}\end{array}\right)$ with

$$
A_{ \pm}=\left\{\left[\operatorname{Im}(a)^{2}+|b|^{2}\right]^{1 / 2} \pm \operatorname{Im}(a)\right\}^{1 / 2}
$$

and

$$
\begin{aligned}
& a=\exp \left[-\frac{1}{2} i T\left(E_{1}-E_{2}\right)\right] ; \\
& b=i \exp \left[-\frac{1}{2} i T\left(E_{1}-E_{2}\right)\right] \mathscr{V}(t) .
\end{aligned}
$$

Equations (46) - (48) may be easily recovered in the following manner. Insert (63) into (62) and (61). Insert (61) and (58) into (53). It should be noted that the integrand in (53) is time independent.

\section{Summary}

In the preceding sections we learned a method for calculating time mean values of observables. We assumed that these observables refer to a quantum mechanical system subjected to time periodic perturbations. In order to prove that the method is indeed valuable in practical calculations, we applied it to Zener's model. This is essentially a system of order 2 admitting exact solutions for various interesting physical situations. We found the following surprising property of this method. The lowest order calculation (described in the preceding section) yields exact results. This lets us hope that the application of the method developed in this paper should provide very good results also in a more complex physical situation. 
[1] G. Floquet, Ann. Ecole Norm. Sup. Paris 12, 47 (1883).
[2] L. Cesari, Asymptotic Behaviour and Stability Problems in Ordinary Differential Equations, Springer-Verlag, Berlin 1971.

[3] E. A. Coddington and N. Levinson, Theory of Differential Equations, McGraw-Hill, London 1955.
[4] L. Cesari, Mem. Acad. Italia 11, 633 (1941).

[5] A. Messiah, Quantum Mechanics, Vol. I and II, NorthHolland Publishing Company, Amsterdam 1970.

[6] F. J. Dyson, Phys. Rev. 75, 486 (1949).

[7] C. Zener, Proc. Roy. Soc. London A 137, 696 (1932). 